Check for updates

Cite this: RSC Adv., 2018, 8, 16549

\title{
Electrically and electrohydrodynamically driven phase transition and structural color switching of oligomer tethered 2D colloid $\dagger$
}

\author{
Aurangzeb Rashid Masud, ${ }^{a}$ Seung-Ho Hong, ${ }^{b}$ Tian-Zi Shen, ${ }^{a}$ Amir Shahzad ${ }^{a}$ \\ and Jang-Kun Song (iD *a
}

Two-dimensional (2D) nanoparticles in an oligomer-tethered alpha zirconium phosphate ( $\alpha$ ZrP) colloid self-assemble to form a cofacial lamellar structure with regular spacing parallel to the surface and exhibit high reflectance and vivid structural colors within the visible frequency spectrum. Here, we demonstrate electrical switching of the structural color reflection by electrical control of the liquid crystalline phase of the $\alpha \mathrm{ZrP}$ colloid. At low frequency (less than $15 \mathrm{~Hz}$, optimally at $1 \mathrm{~Hz}$ ), electrohydrodynamic flow in the colloid destroys the photonic crystalline lamellar phase and creates an apparently disordered dynamic state with local nematic orientation. The method using electrohydrodynamic flow is a better approach to erase the photonic crystalline ordering of nanoparticles, than application of a high-frequency field, which has been proposed previously, in terms of the required voltage and color uniformity. The field-induced disordered particle orientation can be spontaneously recovered to the initial photonic crystal state by removing the applied voltage, but this method requires quite a long time and does not work in materials with a high nanoplatelet concentration. On the other hand, by applying a horizontal high-frequency field (approximately $10 \mathrm{kHz}$ ), the initial lamellar ordering can be forcibly recovered. In this way, the structural color in the 2D nanoparticle colloid can be repeatedly erased or rewritten by switching the frequency of the applied voltage from $10 \mathrm{kHz}$ to $1 \mathrm{~Hz}$ and vice versa, respectively. Our method of switching a 2D colloid using both electrohydrodynamic flow and frequency modulation is expected to be a promising approach to control the photonic crystallinity of colloidal photonic crystals.

\author{
Received 13th March 2018 \\ Accepted 30th April 2018 \\ DOI: $10.1039 / c 8 \mathrm{ra02186d}$ \\ rsc.li/rsc-advances
}

\section{Introduction}

Structural color reflection from photonic crystals is an interesting phenomenon that occurs when electromagnetic waves in the visible spectral range interact with nanostructured materials having a periodic modulation in refractive index. ${ }^{1,2}$ The coherent interference of scattered light from these materials produces a secondary emission at regular angles commonly termed as Bragg's optical diffraction. A wide range of optical and photonic applications based on the structural color reflection and the stop-band features of the photonic crystal materials have been proposed. For instance, dynamic lyotropic pitch tuning of the periodic phases or photo patterning the colloidal crystals can be used for color-tunable functional films, ${ }^{3,4}$ lowpower consumption reflective color displays, ${ }^{5-7}$ colorimetric chemical sensors and iridescent pigments. $^{8-10}$ Recently,

${ }^{a}$ School of Electronics \& Electrical Engineering, Sungkyunkwan University, Suwon, Gyeonggi-do 16419, South Korea. E-mail: jk.song@skku.edu

${ }^{b}$ College of Information \& Communication Engineering, Sungkyunkwan University, Suwon, Gyeonggi-do 16419, South Korea

$\dagger$ Electronic supplementary information (ESI) available. See DOI: $10.1039 / \mathrm{c} 8 \mathrm{ra02186 \textrm {d }}$ colloidal dispersions of $1 \mathrm{~nm}$-thin 2-dimensional (2D) particles have attracted interest owing to its excellent photonic crystallinity and easy manipulation based on its fluidic nature. ${ }^{11,12}$

Reflective color display is a promising candidate for the applications using photonic crystal colloid because of its high demand in portable electronic devices used outdoor, especially in sunlight, where the performance of usual display devices is significantly reduced. Photonic crystal devices retain bright and vivid colors in ambient circumstance, hence, can overcome the poor visibility issue of conventional displays under bright circumstance. For such applications, the tunability of the photonic crystals is essential, and it can be achieved by using external stimuli such as electrical, ${ }^{3,5,12}$ mechanical, ${ }^{13}$ thermal, chemical, and magnetic stimuli. ${ }^{14}$ Among these, the application of electric fields may be the most appropriate and feasible approach to manipulate the local lattice constants of photonic crystals for practical applications such as color displays with numerous pixels. ${ }^{12,15}$ Although the concept of electrical switching of photonic crystallinity in 2D colloids is rather simple, its actual accomplishment is still a great challenge. Color tuning in polymerized colloidal photonic crystals containing silica or polymer spheres was demonstrated based on electrically driven swelling and deswelling. ${ }^{5}$ Similar concept was applied for 
inverse opal structure. ${ }^{6}$ In these methods, only the peak wavelength is tunable, but not the reflectance. Hong et al. demonstrated the erasing and rewriting of color in aqueous 2D graphene oxide (GO) dispersion, based on field-induced reorientation of $2 \mathrm{D}$ particles. ${ }^{\mathbf{1 2}}$ Although the approach works well in tunable photonic devices, intrinsic limitations associated to GO material such as its very low reflectance and poor chemical stability makes it impractical to use the method in reflective display applications. In this way, new materials devoid of these limitations and new switching mechanism are highly demanded.

Alpha zirconium phosphate $(\alpha \mathrm{ZrP})$ is an inorganic $2 \mathrm{D}$ crystal with very high aspect ratio and can form stable colloidal phase upon exfoliation. $\alpha$ ZrP has a number of advantages over other nanocrystalline colloidal structures, including high surface energy, ion exchange capacity, ease intercalation/exfoliation, purity, and controllable surface functionality, and has been widely used in proton conductors in fuel cells, ${ }^{\mathbf{1 6}}$ nanocomposites, ${ }^{17}$ materials for nuclear waste management, ${ }^{18}$ and catalysis. ${ }^{19}$ Recently, it was reported that oligomer tethered $\alpha \mathrm{ZrP}$ colloid can exhibit excellent photonic crystallinity, ${ }^{20}$ where the structural color varies depending on the concentration of $\alpha \mathrm{ZrP}$. However, the electrical switching of $\alpha \mathrm{ZrP}$ photonic crystallinity has not been achieved.

Here we demonstrate the electrical switching of structural color of oligomer tethered $\alpha \mathrm{ZrP}$ colloid that has high structural color reflectance, excellent dispersity and chemical stability. We found that electrohydrodynamic flow can be used to effectively erase the photonic crystal assembly of $\alpha$ ZrP colloid. The erased photonic crystal assembly can be spontaneously recovered with time or electrically reconstructed by applying appropriate electric signals. The results can provide new approach to use $2 \mathrm{D}$ colloid for reflective type displays.

\section{Experimental}

Pristine $\alpha$ - $\mathrm{ZrP}\left(\mathrm{Zr}\left(\mathrm{HPO}_{4}\right)_{2} \cdot \mathrm{H}_{2} \mathrm{O}\right)$ nanocrystals were synthesized via hydrothermal method using $12 \mathrm{M}$ of phosphoric acid and $24 \mathrm{~h}$ reaction time in oven. ${ }^{21} \mathrm{X}$-Ray diffraction (XRD) pattern of the crystals was obtained using D8 Discover (Bruker Instrument, Germany). Sharp peaks of the XRD pattern confirms the high-level crystallinity of the synthesized material as shown in Fig. 1a. Field emission scanning electron microscopic (FESEM) analysis was carried out using JSM-7600F (JEOL, Japan), and revealed that the majority of nanocrystals particles were of irregular hexagonal shapes with the mean diameter of $1300 \pm$ $250 \mathrm{~nm}$ and the mean thickness of $500 \pm 70 \mathrm{~nm}$ (Fig. 1b). These nanocrystals were exfoliated using oligomeric surfactant polyoxyalkyleneamine, with the molecular weight of $1000 \mathrm{~g} \mathrm{~mol}^{-1}$ (Jeffamine M1000, Huntsman Chemicals, USA) ${ }^{20}$ Nanoplatelets of oligomer tethered $\alpha \mathrm{ZrP}$ crystals were obtained in the form of embedded particles within the polymer gel. To obtain the FESEM image of exfoliated particles, low concentration $\alpha \mathrm{ZrP}$ in acetone was spin coated on silicon dioxide wafer at $500 \mathrm{rpm}$ for $30 \mathrm{~s}$, and the thin film was baked at $80{ }^{\circ} \mathrm{C}$ in nitrogenous environment for 1 hour. The FESEM image of the exfoliated particles is shown in Fig. 1c, where dotted lines are drawn to
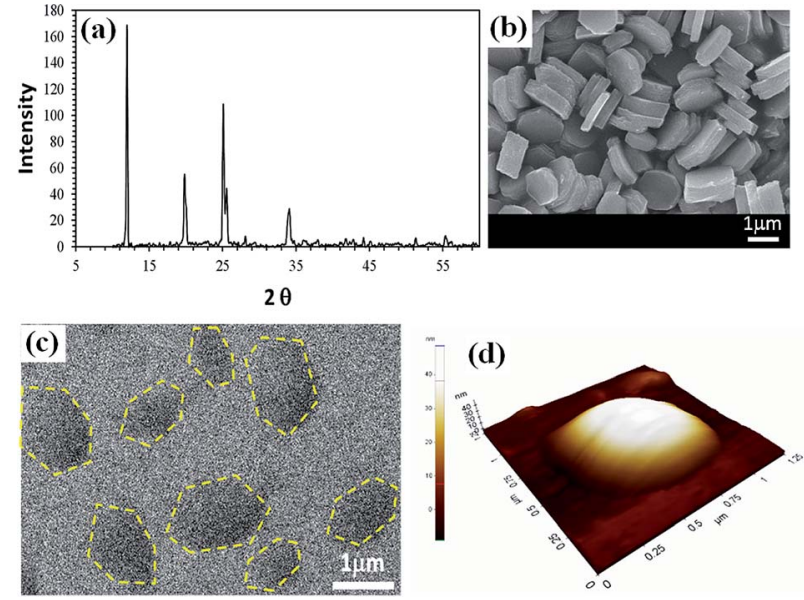

Fig. 1 Characterization of synthesized $\alpha$ ZrP nanocrystals and exfoliated nanoplatelets. (a) The XRD pattern of synthesized $\alpha$ ZrP crystals. (b) The FESEM image shows hexagonal shaped crystals of $\alpha Z r P$ having average diameter of $1300 \pm 250 \mathrm{~nm}$. (c) Exfoliated $\alpha \mathrm{ZrP}$ particles that are tethered with oligomer. (d) AFM image of $(\sim 1 \mathrm{~mm})$ diameter oligomer coated $\alpha$ ZrP nanoplatelet.

elaborate the boundaries of three nanoplatelets on the surface. To approximate the thickness of the swelled exfoliated nanoplatelets, atomic force micrographic (AFM) analysis was performed using PSIA XE-100 (Park Systems, Korea). Fig. 1d show the reconstructed AFM image for a dried oligomer tethered $\alpha \mathrm{ZrP}$ particle. The AFM profile shows a thickness of $48.5 \mathrm{~nm}$ and a diameter of $1 \mu \mathrm{m}$. Zeta potential of exfoliated $\alpha \mathrm{ZrP}$ colloid dispersed in DMF was measured at $0.01 \mathrm{wt} \%$ using dynamic light scattering instrument (Zetasizer Nano, Malvern Instrument Ltd, UK), which was $-45 \mathrm{mV}$. Spectral reflectance was measured using a spectro-photometer, CM-2500d (Konica Minolta, Japan) under specular component excluded (SCE) mode.

To investigate the electro-optical color switching response of oligomer-tethered $\alpha$-ZrP, we made two types of simple cells with different field direction. In the first type of cell (type-A: Fig. 2a), two transparent indium tin oxide (ITO) glass substrates were used as the top and bottom substrates. $\alpha \mathrm{ZrP}$ colloid was filled between the substrates. The field direction is vertical for the type-A cell. In the second type cell (type-B: Fig. 2b), patterned ITO electrodes with interdigitated shapes were used, and either of vertical and horizontal electric field can be applied. The electrode width and inter-electrode gap was fixed to $1 \mathrm{~mm}$ and $1.5 \mathrm{~mm}$. In both cells, polydimethylsiloxane (PDMS) wall spacers were used to hold the sample and to sustain an optical path length of $1.5 \mathrm{~mm}$ between the top and bottom substrates.

\section{Results and discussions}

\section{High quality photonic crystals of $\alpha \mathrm{ZrP}$}

The exfoliated oligomer tethered nanocomposites were dispersed in $N, N$-dimethyl formamide (DMF) at various concentrations from 0.4 to $1.09 \mathrm{wt} \%$. The suspensions exhibited vivid structural colors with very high reflectance in glass bottles under white illumination as shown in the top of Fig. 3, the vivid 


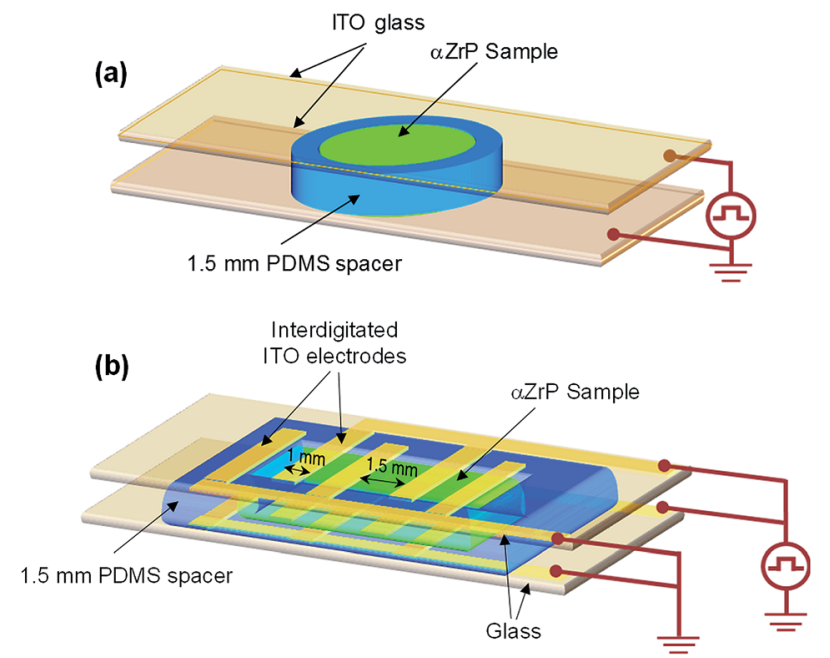

Fig. 2 Schematic of cells. (a) Type-A cell with fully covered ITO glass on top and bottom substrates. (b) Type-B cell with interdigitated electrodes.

structural color reflection implies the periodic lamellar arrangement of $\alpha \mathrm{ZrP}$ nanoparticles in colloid, which is stabilized by both long-range electrostatic repulsion and presence of M1000 oligomer brush layers tethered onto the surface of nanoplatelets. ${ }^{20}$ The nanoplate concentrations corresponding to the red to blue spectral reflectance are $0.4 \mathrm{wt} \%$ to $1.09 \mathrm{wt} \%$, which accords well with the theoretical expectation. The estimated interlayer spacing (d) using Platz equation $\left(d=\lambda_{\max } / 2 n\right)$ exhibit a linear relationship with the inverse volume fraction (1/ $\phi$ ) of $\alpha \mathrm{ZrP}$ colloid in DMF (refractive index $n=1.43$ ) as show in the inset of Fig. 3. The peak reflectance was the highest for the blue $\alpha \mathrm{ZrP}$ colloid, which reaches up to $60 \%$, and it decreases gently with decreasing concentration, and it was about $30 \%$ for the red sample. The peak reflectance reflects the quality of

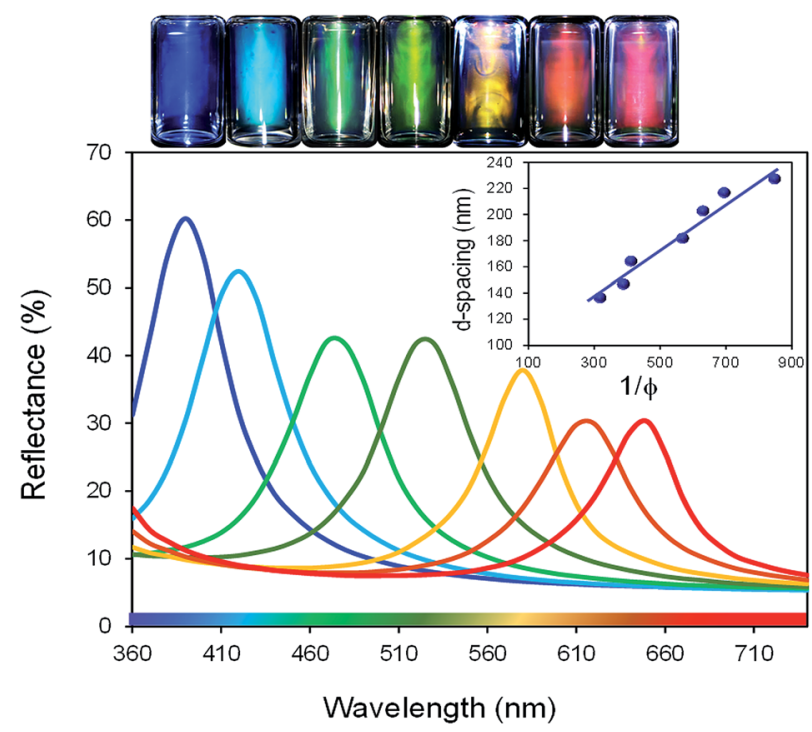

Fig. 3 Bottles of aZrP-DMF dispersions with the concentration from 0.4 to $1.09 \mathrm{wt} \%$, and their spectral reflectance. Inset image shows the linear dependence of inter layer spacing on inverse volume fraction of colloidal concentration. periodicity in the lamellar assembly of nanoparticles, hence the high concentration dispersion has better periodic assembly than that with low concentration, indicating the stronger interparticle interaction in higher concentration colloids.

\section{Structural color switching using vertical electric field in type-A cells}

When the $\alpha \mathrm{ZrP}$ colloids were injected in a type-A cell with flat surfaces, the $\alpha \mathrm{ZrP}$ lamellae were likely to align parallel to the substrates and the cell exhibited the same vivid color reflections, as shown in Fig. 4a. To investigate the electric field on the nanoparticle alignment, we firstly applied $10 \mathrm{kHz}$ square electric field across the type-A cell. ${ }^{12}$ The application of high frequency electric fields induces Maxwell-Wagner polarization owing to the vibration of ions around anisotropic $\alpha \mathrm{ZrP}$ particles, and the field-induced polarization is usually parallel to the particle long axis. Hence, the $\alpha \mathrm{ZrP}$ particles are likely to align vertically under the application of $10 \mathrm{kHz}$ electric fields. As shown in Fig. $4 \mathrm{a}$, where the cell contains $0.6 \mathrm{wt} \%$ $\alpha \mathrm{ZrP}$ colloid, the bright green color reflection starts to disappear from the edge of cell at $5 \mathrm{~V}$ application, and completely disappears at $9 \mathrm{~V}$. When the cell was observed under the crossed polarizers, the birefringent pattern is observed (the last two images in Fig. 4a), indicating the particle alignment direction. Owing to the injection flow of $\alpha \mathrm{ZrP}$ colloid (from left to right of the cells in Fig. 4), the initial alignment has significant birefringent flow mark at $0 \mathrm{~V}$. Although the particles near surface are aligned parallel to the substrate, those in the middle of cell may tilt from the surface and produce the birefringence pattern. The 2D particle alignment depending on the depth from the surface was systemically investigated using 2D GO colloid in our previous work. ${ }^{22}$ The application of $9 \mathrm{~V}$ does not alter the shape of pattern, but the brightness is significantly enhanced, as shown in the last image in Fig. 4a. Since the birefringence is maximized when 2D particles align vertically to the substrate, the enhanced brightness indicates the larger tilting angle of particles under the application of electric fields, which proves that the application of $10 \mathrm{kHz}$ electric fields makes $\alpha \mathrm{ZrP}$ particles align vertically while sustaining the cofacial alignment. The tilting of $\alpha \mathrm{ZrP}$ particles makes the lamellar layer stand vertically to the surface and no structural color is observed on top viewing. However, the color removal is not uniform in the cell, but sensitively depends on the initial alignment condition. In this cell, the color disappears from the edge, as well indicated in the second image in Fig. 4a. Another problem is that the voltage required to remove the structural color sensitively depends on the concentration, and the structural color of blue cell was hardly removed even under the application of $20 \mathrm{~V}$ (image not shown here). ${ }^{12}$

In the second experiment, we applied $1 \mathrm{~Hz}$ electric field across the same cell. Three different concentrations with red $(0.4 \mathrm{wt} \%)$, green $(0.6 \mathrm{wt} \%)$ and blue $(0.93 \mathrm{wt} \%)$ color reflections were used for the experiments, as shown in Fig. 4b. The application of $1 \mathrm{~Hz}$ electric fields also removed the structural color reflection for all the samples as shown in Fig. $4 \mathrm{~b}$. The structural color became weaker in the whole area of the cell, as the applied 
(a) $10 \mathrm{kHz}$
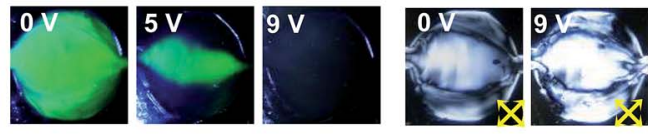

(b) $\frac{1 \mathrm{H}}{\mathrm{O}}$
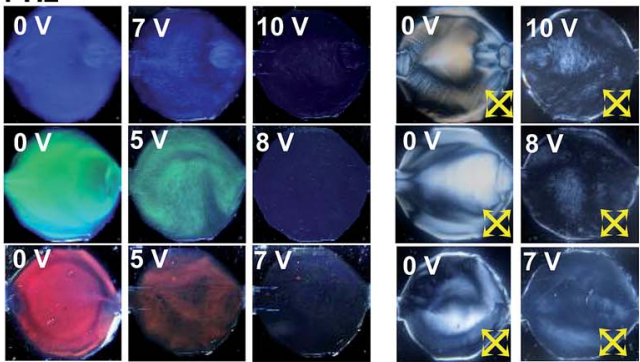

(d)
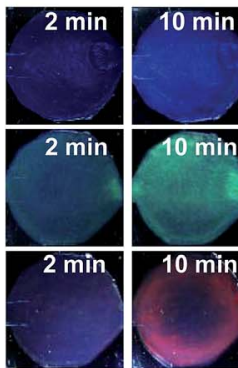

$30 \mathrm{~min}$
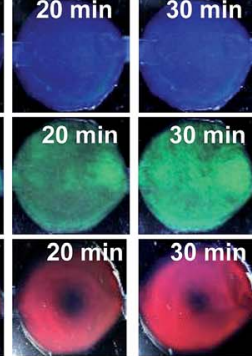

(c)

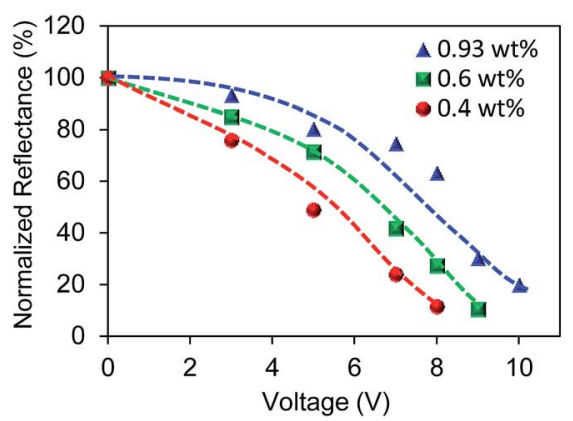

(e)

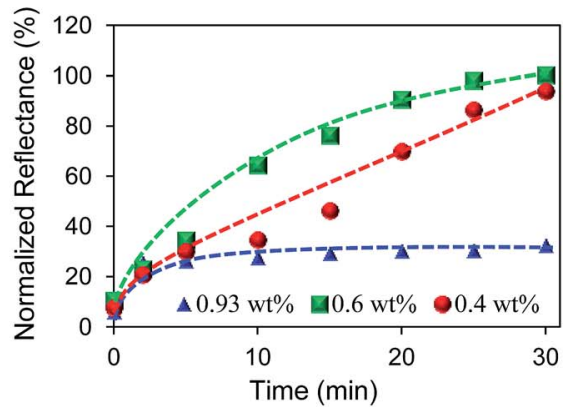

Fig. 4 (a) Application of $10 \mathrm{kHz}$ vertical electric field removes the structural color in $\alpha \mathrm{ZrP}$ colloid (left three images), and enhances the birefringence (right two images). (b) The application of $1 \mathrm{~Hz}$ vertical electric field also removes the structural colors of $\alpha \mathrm{ZrP}$ colloid, but the birefringence is reduced by the $1 \mathrm{~Hz}$ field, differently from $10 \mathrm{kHz}$ field applications. Concentrations: 0.93, 0.6, and 0.4 for blue, green, and red colloids. (c) Increasing reflectance as a function of applied voltage. (d) Spontaneous recovery of structural color with time. (e) Increasing reflectance with time during the spontaneous recovery of structural color.

voltage increased. Although the voltage required to remove the structure color depends on the concentration, the difference was smaller than that in the high frequency experiment; it was $7 \mathrm{~V}$ for red colloid and was $10 \mathrm{~V}$ for blue one. Thus, in terms of the color uniformity and the concentration dependency on the applied voltage, the use of low frequency electric field was a better approach to remove the structural color.

The underlying mechanism was also different from that in the high frequency case. Under the application of low frequency electric fields (below $\sim 15 \mathrm{~Hz}$ ), electrohydrodynamic flow was observed, and the dynamic flow disturbs the lamellar assembly of $\alpha \mathrm{ZrP}$ particles, erasing the structural color reflection in the cell. The birefringent patterns under the low frequency electric fields were also different from that under the high frequency electric fields, as shown in the last two columns in Fig. 4b. Under $1 \mathrm{~Hz}$ electric signals, dark state was observed, differently from the bright vertical assembly under $10 \mathrm{kHz}$ fields. This indicates that the dynamic flow disturbs the nematic ordering and decreases the ordering of particles. The dynamic flow is well discernible in the Video M-1 in the ESI $\dagger$ under $1 \mathrm{~Hz}$ electric fields.

Fig. 4c shows the decreasing peak reflectance with increasing applied voltage during the $1 \mathrm{~Hz}$ electric switching experiment. All the samples exhibit a similar trend with increasing voltage, but the sensitivity on electric voltage depends on the concentration. An increase in colloidal concentration reduces the interparticle and interlamellar distances and the interparticle and interlayer interactions (electrostatic and steric repulsive forces), as well, resulting in the slight desensitization of electrical switching.

After obtaining the filed-induced dark state, the electric field was removed to allow the spontaneous recovery of structural colors. For the red and green color cells with relatively low concentrations, the structural color reflection was almost recovered after $30 \mathrm{~min}$, as shown in Fig. $4 \mathrm{~d}$ and e. On the other hand, for the high concentration cell with blue color, the structural color was not fully recovered, but only $30 \%$ of reflectance was obtained after $30 \mathrm{~min}$. The slow and poor recovery of structural color in the high concentration cell is due to the high packing ratio and high inter-particle friction. ${ }^{12}$ Interestingly, the spontaneous recovery of photonic crystal was relatively weakly observed after applying high frequency electric field to induced vertical alignment, as shown in Fig. S1 in ESI. $\dagger$ That is, the planar lamellar structure is spontaneously recovered from disordered state, but is not spontaneously obtained from vertically aligned dark state.

In this way, the electrohydrodynamic migration of nanoparticles can be an effective way to remove the structural color reflection in photonic crystalline aZrP colloid. However, the spontaneous recovery of photonic crystallinity from the erased cell is not an appropriate method owing to its slow response and incomplete recovery. Hence, a forced recovery method may be necessary, and such a forced recovery is not possible in the typeA cell with vertical fields. 


\section{Structural color switching using vertical electric field in type-B cells}

To improve the dynamic response of structural color switching, the type-B cells were used. The electric field direction in the type-B cells can be switchable between vertical and horizontal directions by applying appropriate electric signals. In the first experiment using the type-B cell, vertical electric field was used as shown in Fig. 5a, where the electrodes in the top substrate was applied by $10 \mathrm{~V}$ and $10 \mathrm{kHz}$ or $1 \mathrm{~Hz}$ square electric fields, and the electrodes in the bottom substrate was applied by ground signals. Injecting $0.6 \mathrm{wt} \% \alpha \mathrm{ZrP}$ colloid in the type-B cell, the cell exhibits green color reflection as shown in Fig. 5a. When applying vertical electric fields of $10 \mathrm{kHz}$ or $1 \mathrm{~Hz}$, the structural color in the areas of ITO electrodes disappeared (Fig. 5a). As explained in the previous section, $\alpha \mathrm{ZrP}$ particles align vertically under the high frequency fields and the lamellar assembly is destroyed under the low frequency electric fields. Both method effectively remove the structural color reflection. On the other hand, the color reflection between two neighbouring electrodes is not influenced by the vertical fields, because the electric fields in the region is too weak to alter the initial lamellar structure (see the right images in Fig. 5a). The nanoparticle orientations on electrodes and between two adjacent electrodes are shown in the schematics in Fig. 5(a-i) and (a-ii). In case of the application of $10 \mathrm{kHz}$ electric fields, the nanoparticles in the high field region align vertically, but those in between adjacent electrodes remain parallel to the glass substrate. In case of the application of $1 \mathrm{~Hz}$ fields, the nanoparticles in the high field region are randomly distributed.

\section{Structural color switching using frequency-modulated horizontal electric field in type-B cells}

In the next experiment using the type-B cell, a horizontal electric field was applied, as illustrated in the right top of Fig. 5b-i. Initially, the cell exhibited uniform green color reflection (Fig. 5b-i), and the cell had random birefringence pattern under the crossed polarizers. Then, when $1 \mathrm{~Hz} 10 \mathrm{~V}$ square electric field was applied horizontally, the color reflection disappeared in the whole area of the cell (Fig. 5b-ii). Microscopic observation in Fig. 5c shows hydrodynamic oscillatory flow of colloid, responding to the $1 \mathrm{~Hz}$ signals. The group particles in the marked area oscillates rightward and leftward following the direction of electric field. The oscillatory particle motion is well discernible when adding small quantity of carbon nanotube particles (CNT) in colloid, as shown in Fig. S2 in ESI. $\dagger$ Such hydrodynamic flow continuously disturbs the photonic crystalline assembly of nanoparticles. The birefringence pattern was weaker and dynamically altered with time (right images of Fig. 5b-ii). Under such dynamic state, the highly ordered particle arrangement for Bragg's reflection cannot persist, but the ordering state keeps changing. The origin of the electrohydrodynamic flow could be due to the field induced electroosmotic flow in the colloid. ${ }^{23,24}$

Upon changing the field frequency to $10 \mathrm{kHz}$ at the same voltage, the structural color reflection reappeared between the adjacent electrodes, where horizontal electric fields are applied. On the other hand, the region on each electrode does not recover the initial green color, because of weak electric field in the region. Video clip M-2 in the ESI $\dagger$ clearly shows the color removal and reappearance between electrodes of the cell when $(\mathrm{a}-\mathrm{i})$

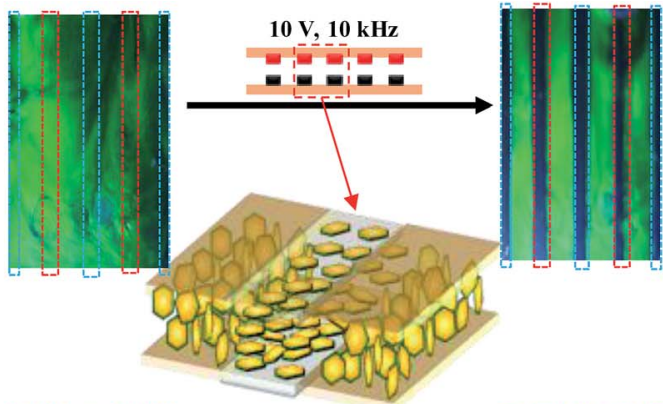

(a-ii)

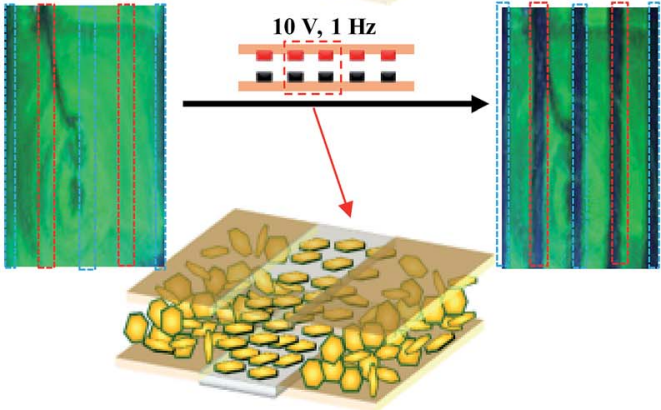

(b-i)
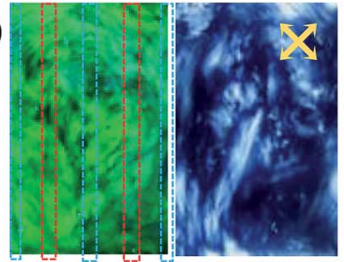

(b-ii)
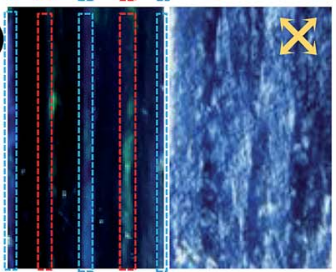

(b-iii)

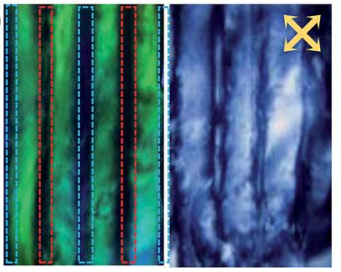

$10 \mathrm{~V}, 1 \mathrm{~Hz} / 10 \mathrm{kHz}$

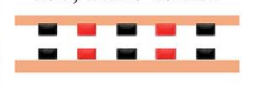

(c)

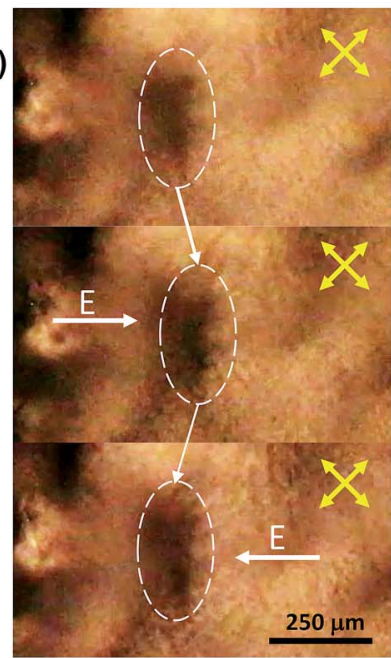

Fig. 5 (a) (i) $10 \mathrm{kHz}$ (ii) $1 \mathrm{~Hz}$ vertically applied electric field, electrode configuration (Black = GND and Red = signal) and particles orientation within two adjacent electrodes. Red and blue dotted lines in cell represent the location of ground and signal electrodes respectively. (b) (i) Initial state of cell with electrodes configuration for horizontal field signal, horizontal field induced color variations at (ii) $V=10 \mathrm{~V}, f=1 \mathrm{~Hz}$ (iii) $V=10 \mathrm{~V}, f$ $=10 \mathrm{kHz}$ and corresponding birefringence pattern. (c) Dynamic state oscillatory motion of $\alpha \mathrm{ZrP}$ colloid when subjected to $1 \mathrm{~Hz}$ electric field. Group of particles in marked area is oscillating right and left along the field direction. 
(a)

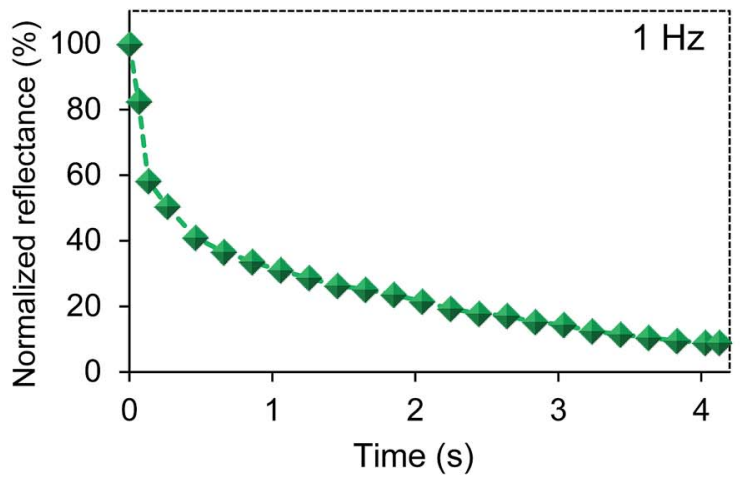

(b)

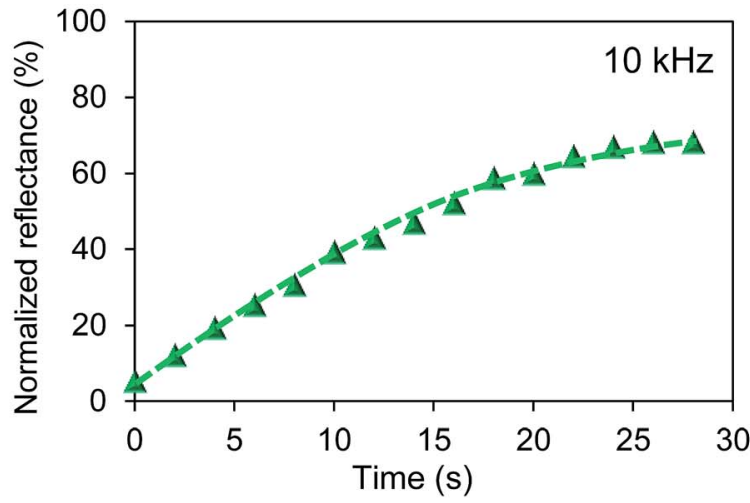

(c)

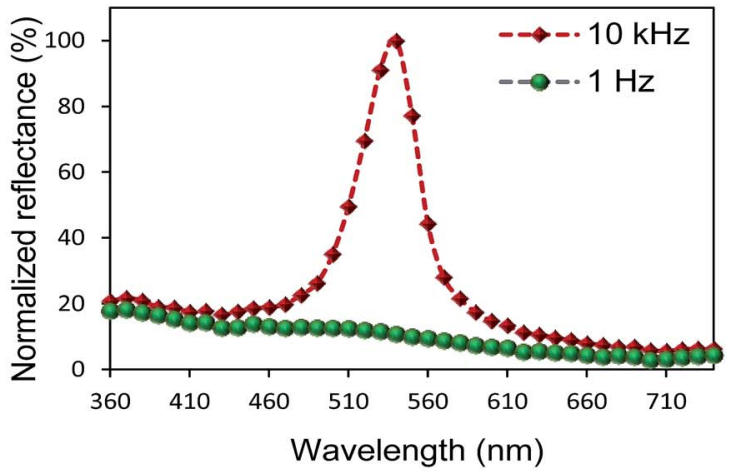

(d)

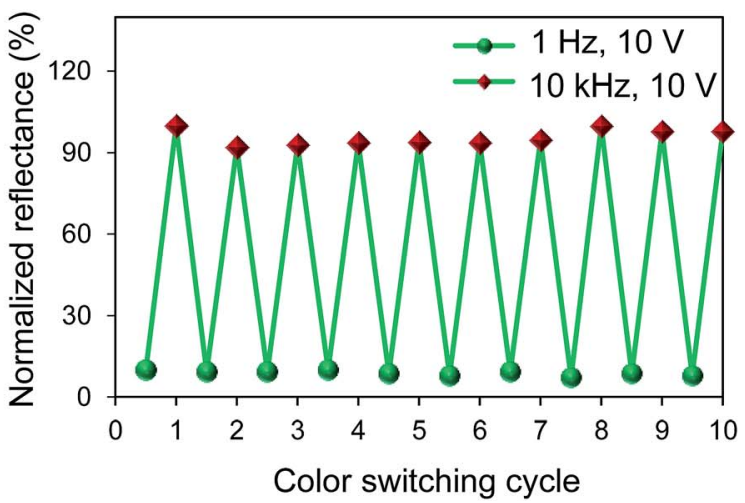

Fig. 6 (a) Normalized reflectance percentage of structural color removal as a function of time. (b) Normalized reflectance percentage of structural color recovery as a function of time. (c) Normalized reflectance spectra of initial state, at $1 \mathrm{~Hz}$ and $10 \mathrm{kHz}$. (d) Normalized reflectance percentage of ten consecutive field induced color switching ON and OFF cycles.

the frequency of electric fields switches from $10 \mathrm{kHz}$ to $1 \mathrm{~Hz}$ and vice versa, respectively. The application of horizontal $10 \mathrm{kHz}$ electric field in between the adjacent electrodes induces not only the photonic crystallinity, which may be mostly influenced by the lamellar alignment of nanoparticles near the surface, but also the optical birefringence, which may be mostly caused by the overall particle alignment in the bulk sample (see the right image of Fig. 5b-iii).

The dynamic response of peak reflectance is shown in Fig. 6a, where the normalized peak reflectance is plotted as a function of time under the application of $1 \mathrm{~Hz} 10 \mathrm{~V}$ horizontal field. The peak reflectance decreases quickly down to $50 \%$ within $0.2 \mathrm{~s}$, and then, the response becomes slower and reaches to about $9 \%$ of initial reflectance after $4 \mathrm{~s}$. When $10 \mathrm{kHz}$ $10 \mathrm{~V}$ electric field was applied, the structural color reflection was recovered within $25 \mathrm{~s}$. The $10 \mathrm{kHz}$ horizontal electric field makes the longest axis of 2D particles align along the field direction, which, however, is not sufficient to induce lamellar alignment of $2 \mathrm{D}$ particles, because of no force to align the second longest axis of 2D particles. Hence, the recovery time is longer than the erasing time of structural color, although the forced recover is much quicker than the spontaneous recovery. The surface effect may help 2D particles align parallel to the substrate. Microscopic observation for the transition between the dynamic state at $1 \mathrm{~Hz}$ and the static state at $10 \mathrm{kHz}$ is shown in the video clip M-3 in the ESI. $\dagger$ Fig. $6 \mathrm{c}$ shows the relative spectral reflectance under the application of $10 \mathrm{kHz}$ and $1 \mathrm{~Hz}$ electric fields.

The erasing and writing of the field-induced photonic crystals were repeatedly obtained by modulating the frequency of the applied electric fields, as shown in Fig. 6d, which shows the good cyclic stability of the switching process in $\alpha \mathrm{ZrP}$ colloid.

\section{Conclusion}

Electrical switching between photonic crystalline lamellar assembly and disordered dynamic state was demonstrated using oligomer tethered $\alpha \mathrm{ZrP}$ colloid. The dynamic state existed under the application of the electric field with the frequencies below $\sim 15 \mathrm{~Hz}$, while the lamellar photonic crystalline phase was induced by applying the fields with above $\sim 500 \mathrm{~Hz}$ (optimally at around $10 \mathrm{kHz}$ ). By switching the frequency of electric fields from $1 \mathrm{~Hz}$ to $10 \mathrm{kHz}$ and vice versa in the cell with interlaced electrodes on both substrates, we could selectively obtain the well-aligned photonic crystalline lamellar phase and practically disordered dynamic state, which exhibits excellent photonic crystal reflection and dark state with no reflection, respectively. In particular, we introduced electrohydrodynamic flow to remove the photonic crystalline assembly in $2 \mathrm{D}$ colloid, which takes just a few seconds to fully erase the photonic crystallinity. Although the disordered dynamic state spontaneously turns into photonic crystalline lamellar state with time, the spontaneous process requires long time up to a half hour. 
On the other hand, forced recovery using $10 \mathrm{kHz}$ horizontal field can reduce the switching time down to 25 seconds, in which the recovery of the cofacial alignment of nanoplatelets near the glass substrate is achieved by the cooperative influence of the electric field effect and the surface field effect. Our results show that the performance of the electrical switching $\alpha \mathrm{ZrP}$ colloid are significantly improved compared to the previous report using 2D GO colloid, in reflectance, switching time, color homogeneity, and switching cycle's stability. Moreover, the iridescent $\alpha \mathrm{ZrP}$ colloid is easily synthesized as compared to the iridescent 2D GO. Compared to tunable photonic crystal using spherical particles, which controls the peak reflectance color, our cell using 2D colloid exhibits tunability in reflectance at the same wavelength. The cell is switchable between complete dark and bright states by controlling the phase of the colloid. In our cell the new method to switch 2D colloid using both electrohydrodynamic flow and electric double layer polarization may be a promising approach to electrically control the photonic crystallinity in colloidal photonic crystals.

\section{Conflicts of interest}

There are no conflicts to declare.

\section{Acknowledgements}

This project was funded by Samsung Research Funding Center of Samsung Electronics Project No. SRFCMA1402-03.

\section{Notes and references}

1 K. Katagiri, Y. Tanaka, K. Uemura, K. Inumaru, T. Seki and Y. Takeoka, Structural Color Coating Films Composed of an Amorphous Array of Colloidal Particles via Electrophoretic Deposition, NPG Asia Mater., 2017, 9(3), 1-4.

2 G. Zyla, A. Kovalev, M. Grafen, E. L. Gurevich, C. Esen, A. Ostendorf and S. Gorb, Generation of Bioinspired Structural Colors via Two-Photon Polymerization, Sci. Rep., 2017, 7(1), 1-9.

3 H. Kim, J. Ge, J. Kim, S. Choi, H. Lee, H. Lee, W. Park, Y. Yin and S. Kwon, Structural Colour Printing Using a Magnetically Tunable and Lithographically Fixable Photonic Crystal, Nat. Photonics, 2009, 3(9), 534-540.

4 S. H. Kim, H. S. Park, J. H. Choi, J. W. Shim and S. M. Yang, Integration of Colloidal Photonic Crystals toward Miniaturized Spectrometers, Adv. Mater., 2010, 22(9), 946950.

5 A. C. Arsenault, D. P. Puzzo, I. Manners and G. A. Ozin, Photonic-Crystal Full-Colour Displays, Nat. Photonics, 2007, 1(8), 468-472.

6 D. P. Puzzo, A. C. Arsenault, I. Manners and G. A. Ozin, Electroactive Inverse Opal: A Single Material for All Colors, Angew. Chem., Int. Ed., 2009, 48(5), 943-947.

7 T. S. Shim, S. H. Kim, J. Y. Sim, J. M. Lim and S. M. Yang, Dynamic Modulation of Photonic Bandgaps in Crystalline Colloidal Arrays under Electric Field, Adv. Mater., 2010, 22(40), 4494-4498.
8 J. H. Holtz and S. A. Asher, Polymerized Colloidal Crystal Hydrogel Films as Intelligent Chemical Sensing Materials, Nature, 1997, 389, 829-832.

9 Y. Zhao, X. Zhao, C. Sun, J. Li, R. Zhu and Z. Gu, Encoded Silica Colloidal Crystal Beads as Supports for Potential Multiplex Immunoassay, Anal. Chem., 2008, 80(5), 15981605.

10 S. H. Kim, S. Y. Lee, G. R. Yi, D. J. Pine and S. M. Yang, Microwave-Assisted Self-Organization of Colloidal Particles in Confining Aqueous Droplets, J. Am. Chem. Soc., 2006, 128(33), 10897-10904.

11 P. Li, M. Wong, X. Zhang, H. Yao, R. Ishige, A. Takahara, M. Miyamoto, R. Nishimura and H. J. Sue, Tunable Lyotropic Photonic Liquid Crystal Based on Graphene Oxide, ACS Photonics, 2014, 1(1), 79-86.

12 S.-H. Hong, T.-Z. Shen and J.-K. Song, Manipulation of Structural Color Reflection in Graphene Oxide Dispersions Using Electric Fields, Opt. Express, 2015, 23(15), 18969.

13 D. Ge, E. Lee, L. Yang, Y. Cho, M. Li, D. S. Gianola and S. Yang, A Robust Smart Window: Reversibly Switching from High Transparency to Angle-Independent Structural Color Display, Adv. Mater., 2015, 27(15), 2489-2495.

14 K. Sano, Y. S. Kim, Y. Ishida, Y. Ebina, T. Sasaki, T. Hikima and T. Aida, Photonic Water Dynamically Responsive to External Stimuli, Nat. Commun., 2016, 7, 1-9.

15 M. G. Han, C. J. Heo, H. Shim, C. G. Shin, S. J. Lim, J. W. Kim, Y. W. Jin and S. Lee, Structural Color Manipulation Using Tunable Photonic Crystals with Enhanced Switching Reliability, Adv. Opt. Mater., 2014, 2(6), 535-541.

16 J. E. Kim, S. B. Park and Y. I. Park, Proton-Conducting Zirconium Phosphate Glass Thin Films, Solid State Ionics, 2012, 216, 15-18.

17 H. Wu, C. Liu, J. Chen, Y. Yang and Y. Chen, Preparation and Characterization of Chitosan/ $\alpha$-Zirconium Phosphate Nanocomposite Films, Polym. Int., 2010, 59(7), 923-930.

18 B. E. Scheetz, D. K. Agrawal, E. Breval and R. Roy, Sodium Zirconium Phosphate (NZP) as a Host Structure for Nuclear Waste Immobilization: A Review, Waste Manag., 1994, 14(6), 489-505.

19 J. Wu, F. Liu, J. Guo, P. Cui, G. Xu and Y. Cheng, Preparation and Electrorheological Characteristics of Uniform Core/shell Structural Particles with Different Polar Molecules Shells, Colloids Surf., A, 2012, 410, 136-143.

20 M. Wong, R. Ishige, T. Hoshino, S. Hawkins, P. Li, A. Takahara and H. J. Sue, Solution Processable Iridescent Self-Assembled Nanoplatelets with Finely Tunable Interlayer Distances Using Charge- and Sterically Stabilizing Oligomeric Polyoxyalkyleneamine Surfactants, Chem. Mater., 2014, 26(4), 1528-1537.

21 L. Sun, W. J. Boo, H.-J. Sue and A. Clearfield, Preparation of $\alpha$-Zirconium Phosphate Nanoplatelets with Wide Variations in Aspect Ratios, New J. Chem., 2007, 31(1), 39.

22 S.-H. Hong, T.-Z. Shen and J.-K. Song, Shear-Induced Assembly of Graphene Oxide Particles into Stripes near Surface, Liq. Cryst., 2018, DOI: 10.1080/ 02678292.2018 .1435829$. 
23 K. Kang and J. K. G. Dhont, Electric-field induced transitions in suspensions of charged colloidal rods, Soft Matter, 2010, 6, 273-286.
24 J. K. G. Dhont and K. Kang, An electric-field induced dynamical state in dispersions of charged colloidal rods, Soft Matter, 2014, 10, 1987-2007. 